\title{
RED NOISE VERSUS PLANETARY INTERPRETATIONS IN THE MICROLENSING EVENT OGLE-2013-BLG-446
}

\author{
E. Bachelet ${ }^{1}$, D. M. Bramich ${ }^{1}$, C. Han $^{2}$, J. Greenhill ${ }^{3}$, R. A. Street $^{4}$, A. Gould ${ }^{5}$, G. D’Ago ${ }^{6,7}$, K. AlSubai ${ }^{1}$, \\ M. DominiK ${ }^{8,60}$, R. Figuera Jaimes ${ }^{8,9}$, K. Horne $^{8}$, M. Hundertmark ${ }^{8}$, N. Kains ${ }^{9}$, C. Snodgrass ${ }^{10,11}$, I. A. Steele ${ }^{12}$, \\ Y. TSAPRAS ${ }^{4,13}$
}

(The RoboNet COLlaboration),

M. D. Albrow ${ }^{14}$, V. Batista ${ }^{5,15}$, J.-P. Beaulieu ${ }^{15}$, D. P. Bennett ${ }^{16}$, S. Brillant ${ }^{17}$, J. A. R. Caldwell ${ }^{18}$, A. Cassan ${ }^{15}$, A. Cole $^{3}$, C. Coutures ${ }^{15}$, S. Dieters ${ }^{3}$, D. Dominis Prester ${ }^{19}$, J. Donatowicz ${ }^{20}$, P. Fouqué ${ }^{21,22}$, K. Hill ${ }^{3}$, J.-B. Marquette ${ }^{15}$, J. Menzies ${ }^{23}$, C. Pere $^{15}$, C. Ranc ${ }^{15}$, J. Wambsganss ${ }^{24}$, D. Warren ${ }^{3}$

(The Planet COllaboration),

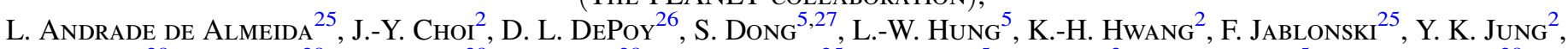

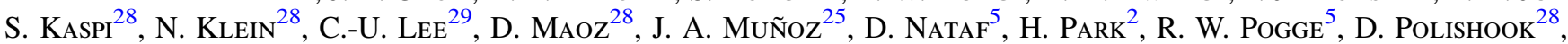
I.-G. SHIN ${ }^{2}$, A. ShPORER ${ }^{30,31}$, J. C. YeE ${ }^{5}$

(THE $\mu$ FUN COLLABORATION),

F. Abe ${ }^{32}$, A. Bhattacharya ${ }^{33}$, I. A. Bond ${ }^{34}$, C. S. Botzler ${ }^{35}$, M. Freeman $^{35}$, A. Fukui ${ }^{36}$, Y. Itow ${ }^{32}$, N. Koshimoto ${ }^{33}$, C. H. Ling $^{34}$, K. Masuda ${ }^{32}$, Y. Matsubara ${ }^{32}$, Y. Muraki ${ }^{32}$, K. Ohnishi ${ }^{37}$, L. C. Philpott ${ }^{38}$, N. Rattenbury ${ }^{38}$, To. Saito ${ }^{39}$, D. J. Sullivan ${ }^{40}$, T. Sumi ${ }^{41}$, D. Suzuki ${ }^{42}$, P. J. Tristram ${ }^{39}$, A. Yonehara ${ }^{40}$

(THE MOA COLlaboration),

AND

V. Bozza ${ }^{43,44}$, S. Calchi Novati ${ }^{43,45,46}$, S. Ciceri ${ }^{47}$, P. Galianni ${ }^{8}$, S.-H. Gu ${ }^{48,49}$, K. HarpsøE ${ }^{50,51}$, T. C. Hinse ${ }^{52}$,

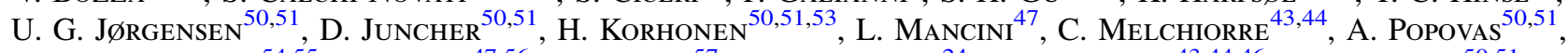
A. Postiglione ${ }^{54,55}$, M. Rabus ${ }^{47,56}$, S. Rahvar ${ }^{57}$, R. W. Schmidt ${ }^{24}$, G. Scarpetta ${ }^{43,44,46}$, J. SkOtTFElt ${ }^{50,51}$, John Southworth ${ }^{58}$, An. Stabile ${ }^{43,44}$, J. Surdej ${ }^{59}$, X.-B. WAng ${ }^{48,49}$, And O. Wertz ${ }^{59}$

(The MiNDSTEP COLlaboration)

${ }^{1}$ Qatar Environment and Energy Research Institute, Qatar Foundation, P.O. Box 5825, Doha, Qatar

${ }^{2}$ Department of Physics, Chungbuk National University, Cheongju 361-763, Korea

${ }^{3}$ School of Mathematics and Physics, University of Tasmania, Private Bag 37, Hobart, TAS 7001, Australia

${ }^{4}$ Las Cumbres Observatory Global Telescope Network, 6740 Cortona Drive, Suite 102, Goleta, CA 93117, USA

${ }^{5}$ Department of Astronomy, Ohio State University, 140 W. 18th Ave., Columbus, OH 43210, USA

${ }^{6}$ Dipartimento di Fisica "E.R. Caianiello," Università di Salerno, Via Ponte Don Melillo, I-84084-Fisciano (SA), Italy Istituto Nazionale di Fisica Nucleare, Sezione di Napoli, Napoli, Italy

${ }^{8}$ SUPA, School of Physics \& Astronomy, University of St Andrews, North Haugh, St Andrews KY16 9SS, UK

${ }_{9}^{9}$ European Southern Observatory, Karl-Schwarzschild-Str. 2, D-85748 Garching bei München, Germany

${ }^{10}$ Max Planck Institute for Solar System Research, Justus-von-Liebig-Weg 3, D-37077 Gttingen, Germany

${ }^{11}$ Planetary and Space Sciences, Department of Physical Sciences, The Open University, Milton Keynes, MK7 6AA, UK

${ }^{12}$ Astrophysics Research Institute, Liverpool John Moores University, Liverpool CH41 1LD, UK

${ }^{13}$ School of Physics and Astronomy, Queen Mary University of London, Mile End Road, London E1 4NS, UK

${ }^{14}$ Department of Physics and Astronomy, University of Canterbury, Private Bag 4800, Christchurch 8020, New Zealand

${ }^{15}$ UPMC-CNRS, UMR 7095, Institut dAstrophysique de Paris, 98bis boulevard Arago, F-75014 Paris, France

${ }^{16}$ Department of Physics, 225 Nieuwland Science Hall, University of Notre Dame, Notre Dame, IN 46556, USA

${ }^{17}$ European Southern Observatory, Casilla 19001, Vitacura 19, Santiago, Chile

${ }_{18}^{18}$ McDonald Observatory, 16120 St. Hwy Spur 78 \#2, Fort Davis, TX 79734, USA

${ }^{19}$ Department of Physics, University of Rijeka, Omladinska 14, 51000 Rijeka, Croatia ${ }^{20}$ Technische Universitat Wien, Wieder Hauptst. 8-10, A-1040 Vienna, Austria

${ }^{21}$ IRAP, CNRS-Université de Toulouse, 14 av. E. Belin, F-31400 Toulouse, France

${ }^{22}$ CFHT Corporation 65-1238 Mamalahoa Hwy Kamuela, Hawaii 96743, USA

${ }^{23}$ South African Astronomical Observatory, P.O. Box 9, Observatory 7935, South Africa

${ }^{24}$ Astronomisches Rechen-Institut (ARI), Zentrum für Astronomie der Universität Heidelberg (ZAH), Mönchhofstr. 12-14, D-69120 Heidelberg, Germany

${ }^{25}$ Departamento de Astronomía y Astrofísica, Universidad de Valencia, E-46100 Burjassot, Valencia, Spain

${ }^{26}$ Department of Physics and Astronomy, Texas A\&M University, College Station, TX 77843-4242, USA ${ }^{27}$ Institute for Advanced Study, Einstein Drive, Princeton, NJ 08540, USA

${ }^{28}$ School of Physics and Astronomy and Wise Observatory, Tel-Aviv University, Tel-Aviv 69978, Israel

${ }^{29}$ Korea Astronomy and Space Science Institute, 776 Daedukdae-ro, Yuseong-gu, Daejeon 305-348, Korea

${ }^{30}$ Las Cumbres Observatory Global Telescope Network, 6740B Cortona Dr, Goleta, CA 93117, USA

${ }^{31}$ Department of Physics, University of California, Santa Barbara, CA 93106, USA

${ }^{32}$ Department of Physics, University of Auckland, Private Bag 92019, Auckland, New Zealand; c.botzler@auckland.ac.nz, p.yock@auckland.ac.nz

Department of Physics, University of Notre Dame, Notre Dame, IN 46556, USA; bennett@nd.edu

${ }^{34}$ Solar-Terrestrial Environment Laboratory, Nagoya University, Nagoya 464-8601, Japan; abe@ stelab.nagoya-u.ac.jp, furusawa@ stelab.nagoya-u.ac.jp,

itow@ @stelab.nagoya-u.ac.jp, kmasuda@ @telab.nagoya-u.ac.jp, ymatsu@ stelab.nagoya-u.ac.jp
35 Okayama Astrophysical Observatory, National Astronomical Observatory of Japan, 3037-5 Honjo, Kamogata, Asakuchi, Okayama 719-0232, Japan

${ }^{36}$ Nagano National College of Technology, Nagano 381-8550, Japan

${ }^{37}$ Tokyo Metropolitan College of Aeronautics, Tokyo 116-8523, Japan

${ }^{38}$ School of Chemical and Physical Sciences, Victoria University, Wellington, New Zealand

${ }^{39}$ Mt. John University Observatory, P.O. Box 56, Lake Tekapo 8770, New Zealand

${ }^{40}$ Department of Physics, Faculty of Science, Kyoto Sangyo University, 603-8555 Kyoto, Japan 


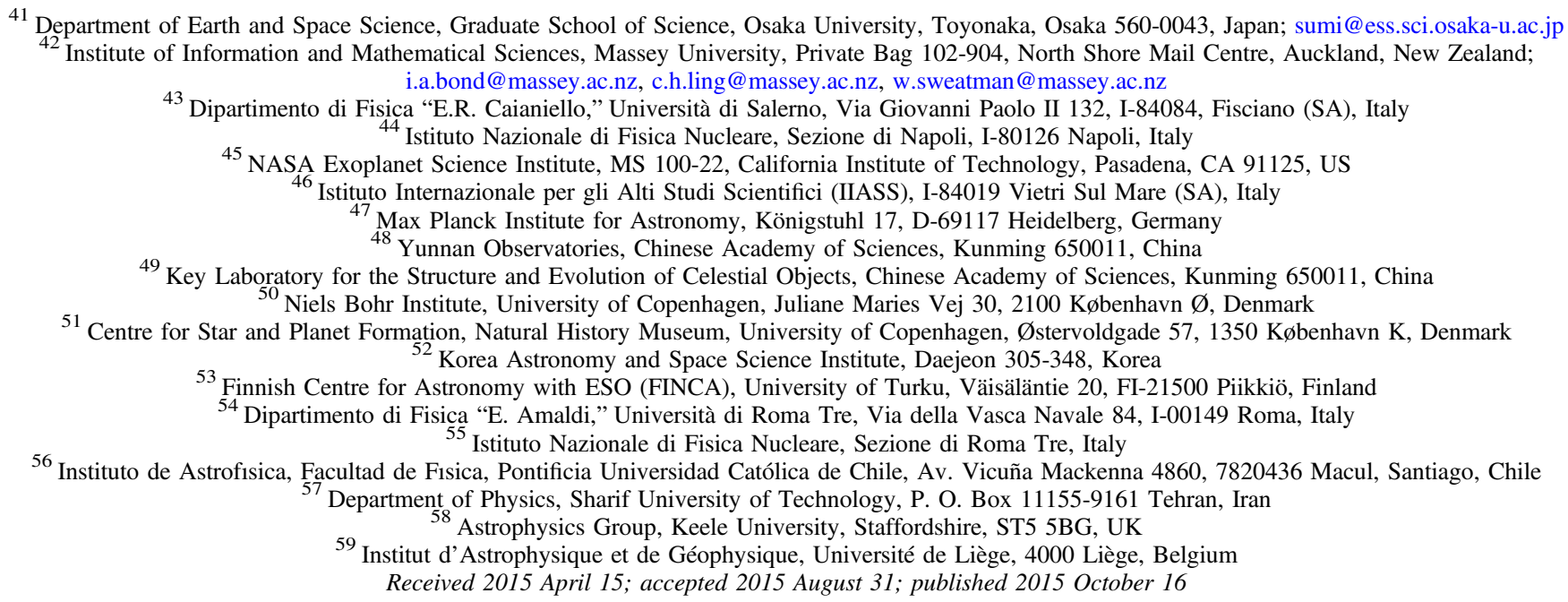

\section{ABSTRACT}

For all exoplanet candidates, the reliability of a claimed detection needs to be assessed through a careful study of systematic errors in the data to minimize the false positives rate. We present a method to investigate such systematics in microlensing data sets using the microlensing event OGLE-2013-BLG-0446 as a case study. The event was observed from multiple sites around the world and its high magnification $\left(A_{\max } \sim 3000\right)$ allowed us to investigate the effects of terrestrial and annual parallax. Real-time modeling of the event while it was still ongoing suggested the presence of an extremely low-mass companion $\left(\sim 3 M_{\oplus}\right)$ to the lensing star, leading to substantial follow-up coverage of the light curve. We test and compare different models for the light curve and conclude that the data do not favor the planetary interpretation when systematic errors are taken into account.

Key words: gravitational lensing: micro - planetary systems - techniques: photometric

\section{INTRODUCTION}

For the past 10 years, gravitational microlensing has been used to detect cool planets around G, K, and M-stars in the Milky Way, allowing access to a planetary regime difficult to observe with the transit or radial velocity methods (i.e., microlensing is sensitive to planets beyond the snowline). Due to the increased field of view of the OGLE-IV and MOA-II surveys, and the recently improved performance of follow-up teams, the number of planets detected by microlensing has gone up substantially (typically 10-20 planets detected per year and 33 published to date).

Another advantage of the microlensing method is that detection of planetary companions is possible over a larger mass range $\left[\sim 1 M_{\oplus}, \sim 13 M_{J}\right]$, including brown dwarfs, if the projected orbital radius $s$ is in the range $0.6-1.6 R_{\mathrm{E}}$ (i.e., the classical "lensing zone"). Thanks to better photometric coverage of light curves, recent studies have advanced claims about the detection of small planets (Bennett et al. 2014). However, smaller mass ratios tend to produce smaller deviations from a single lens model most of the time. Failing to account for photometric systematics can potentially lead to false detections. The analysis of photometric systematics has been important in transit searches and has substantially improved the reliability of detections (Kovács et al. 2005; Smith et al. 2012). This point is too often neglected by the microlensing community.

In this work, we present an extensive study of photometric systematics for the case of OGLE-2013-BLG-0446 and we compare the significance when different microlensing

\footnotetext{
${ }^{60}$ Royal Society University Research Fellow.
}

models are considered. Section 2 presents a summary of the observations of microlensing event OGLE-2013-BLG-0446 from multiple sites around the world. We present our modeling process in Section 3 and conduct a study of systematics in the data in Section 4. We present our conclusions in Section 5 .

\section{OBSERVATIONS}

Microlensing event OGLE-2013-BLG-0446 $\left(\alpha=18^{\mathrm{h}} 06^{\mathrm{m}}\right.$ $56^{\mathrm{s}} 18, \delta=-31^{\circ} 39^{\prime} 27 ! \prime 2$ (J2000.0); $\left.l=0.049, b=-5^{\circ} 344\right)$ was discovered on 2013 April 6 by the Optical Gravitational Lens Experiment (OGLE) Early Warning System (Udalski 2003b) and later alerted by the Microlensing Observations in Astrophysics (MOA) (Bond et al. 2001). Observations obtained on the rising part of the light curve indicated that this event could be highly magnified and might therefore be highly sensitive to planets (Griest \& Safizadeh 1998; Gould et al. 2009; Yee et al. 2009). Follow-up teams, such as $\mu$ FUN (Gould et al. 2006), PLANET (Beaulieu et al. 2006), RoboNet (Tsapras et al. 2009), and MiNDSTEp (Dominik et al. 2008), then began observations a few days before the peak of the event. The peak magnification was $\sim 3000$ and the peak was densely sampled from different observatories.

The various teams used difference image analysis (DIA) to obtain photometry: $\mu \mathrm{FUN}$ used pySIS (Albrow et al. 2009), with the exception of the Auckland data, which were re-reduced using DanDIA (Bramich 2008; Bramich et al. 2013). DanDIA was also used to reduce the RoboNet and the Danish data sets. PLANET data were reduced online with the WISIS pipeline, and final data sets were 
Table 1

Summary of Observations

\begin{tabular}{|c|c|c|c|c|c|c|c|c|}
\hline Name & Collaboration & Location & Aperture(m) & Filter & Code & $N_{\text {data }}$ & Longitude(deg) & Latitude(deg) \\
\hline OGLE_I & OGLE & Chile & 1.3 & $I$ & Woźniak & 463 & 289.307 & -29.015 \\
\hline OGLE_V & OGLE & Chile & 1.3 & $V$ & Woźniak & 24 & 289.307 & -29.015 \\
\hline Canopus_I & PLANET & Tasmania & 1.0 & $I$ & pySIS & 132 & 147.433 & -42.848 \\
\hline Auckland_R & $\mu \mathrm{FUN}$ & New Zealand & 0.4 & $R$ & DanDIA & 107 & 147.777 & -36.906 \\
\hline LSCB_i & RoboNet & Chile & 1.0 & SDSS $-i$ & DanDIA & 378 & 289.195 & -30.167 \\
\hline LSCA_i & RoboNet & Chile & 1.0 & SDSS- $i$ & DanDIA & 385 & 289.195 & -30.167 \\
\hline CPTA_i & RoboNet & South Africa & 1.0 & SDSS $-i$ & DanDIA & 22 & 20.810 & -32.347 \\
\hline CTIO_I & $\mu \mathrm{FUN}$ & Chile & 1.3 & $I$ & pySIS & 112 & 289.196 & -30.169 \\
\hline CTIO_V & $\mu \mathrm{FUN}$ & Chile & 1.3 & $V$ & pySIS & 13 & 289.196 & -30.169 \\
\hline Danish_z & MiNDSTEp & Chile & 1.5 & $i+z$ & DanDIA & 452 & 289.261 & -29.255 \\
\hline MOA_Red & MOA & New Zealand & 1.8 & Red & Bond & 454 & 170.464 & -43.987 \\
\hline Possum_N & $\mu \mathrm{FUN}$ & New Zealand & 0.4 & $N$ & pySIS & 244 & 177.856 & -38.623 \\
\hline Salerno_I & MiNDSTEp & Italy & 0.4 & $I$ & pySIS & 20 & 14.799 & 40.772 \\
\hline Turitea_R & $\mu \mathrm{FUN}$ & New Zealand & 0.4 & $R$ & pySIS & 31 & 175.630 & -40.353 \\
\hline Weizmann_I & $\mu \mathrm{FUN}$ & Israel & 0.4 & $I$ & pySIS & 60 & 34.811 & 31.908 \\
\hline SAAO_I & PLANET & South Africa & 1.0 & $I$ & pySIS & 58 & 20.789 & -32.374 \\
\hline
\end{tabular}

Note. $N$ is unfiltered data set

prepared using pySIS. ${ }^{61}$ OGLE (Udalski \& Szymański 2015) and MOA (Bond et al. 2001) used their own DIA code to reduce their frames. All other data sets were reduced using pySIS.

A total of 2955 data points from 16 telescopes were used for our analysis, after problematic data points were masked. A summary of each data set is available in Table 1 .

\section{MODELING}

\subsection{Source Properties}

This event shows clear signs of finite-source effects and the limb darkening coefficients must be evaluated for each data set. We first consider a point-source point-lens model (PSPL) (Paczyński 1986). The PSPL model allows the estimation of the source and blended fluxes in the $V$ and $I$ passbands for the calibrated OGLE photometry, leading to a good approximation for the $V$ and $I$ magnitudes of the source, which in turn allows us to derive a rough color for the source. We found $(I,(V-I))_{\mathrm{PSPL}}=(19.07,1.48)$. Using the Interstellar Extinction Calculator on the OGLE website ${ }^{62}$ based on Nataf et al. (2013), we found that the Galactic Bulge true distance modulus for this line of sight is $\mu=14.578 \pm 0.326 \mathrm{mag}$ $\left(d_{\text {Bulge }}=8.2 \pm 1.2 \mathrm{kpc}\right)$, the $I$ band extinction is $A_{I}=0.804 \mathrm{mag}$, and the reddening is $E(V-I)=0.683 \pm 0.036 \mathrm{mag}$, leading to $R_{I}=A_{I} / E(V-I)=1.177$, lower than the standard value of 1.5. This low extinction is known as the anomalous extinction law toward the Galactic Bulge; see Udalski (2003a). We derive the source properties as follows:

1. Assuming that the source suffers the same extinction as the Red Giant Clump (i.e., the source is at the same distance), we have $M_{I}=19.07-0.804-14.578=$ $3.7 \mathrm{mag}$, so the source star is most likely a main sequence star. We adopt $\log g \sim 4.5$.

\footnotetext{
61 Data from Tasmania were obtained at the Canopus $1 \mathrm{~m}$ observatory by John Greenhill. This was the last planetary candidate observed from Canopus before its decommissioning. These observations were also the last collected and reduced by John Greenhill (at the age 80 ). He has been our loyal collaborator and friend over the past 18 years and passed away on 2014 September 28.

62 http://ogle.astrouw.edu.pl/
}

2. We derive its effective temperature using the dereddened color-magnitude relation for dwarfs and subgiants (relation (3) in Casagrande et al. 2010) with solar metallicity.

3. From Claret (2000) and using $\log g \sim 4.5$, we are able to find the linear limb-darkening coefficients $u_{\lambda}$ (Milne 1921) for each filter. Following Albrow et al. (1999) and Yoo et al. (2004), we use the transformation:

$$
\Gamma_{\lambda}=\frac{2 u_{\lambda}}{3-u_{\lambda}}
$$

These calculations form the starting point for an iterative fit of the finite source point lens (FSPL) model, together with error-bar rescaling as described in Section 3.3. Our best FSPL model converges to source magnitude and color $(I,(V-I))=(19.00,1.49)$. Correcting for extinction and reddening we have $\left(I_{o},(V-I)_{o}\right)=(18.20,0.81)$. The corresponding effective temperature of the source is $T_{\text {eff }} \sim$ $5400 \mathrm{~K}$, leading to $\Gamma_{V}=0.63\left(u_{V}=0.72\right), \Gamma_{R}=0.55$ $\left(u_{R}=0.65\right)$ and $\Gamma_{I}=0.46\left(u_{I}=0.56\right)$ for $\log g \sim 4.5$. Note that we also use $\Gamma_{I}=0.46$ for the RoboNet telescopes (SDSS- $i$ filter). Finally, given the dereddened magnitude and color of the source from our best FSPL model, we are able to estimate the angular source star radius $\theta_{*}$ using Kervella \& Fouqué (2008):

$$
\begin{aligned}
\log _{10}\left(\theta_{*}\right)= & 3.1982+0.4895(V-I)_{o} \\
& -0.0657(V-I)_{o}^{2}-0.2 I_{o} .
\end{aligned}
$$

The uncertainty of this relation is 0.0238 . The errors on our magnitude estimates are $(\Delta I, \Delta V)=(0.02,0.02)$ mag. Assuming a conservative estimate of the error on $A_{I}$ $(0.1 \mathrm{mag})$ and using standard error propagation gives $9 \%$ precision: $\theta_{*}=0.82 \pm 0.07 \mu$ as. With the adopted source distance $(8.234 \mathrm{kpc})$, the source star radius is $R_{*}=1.4 \pm$ $0.3 R_{\odot}$. Therefore the source is a G6 or K0 star (Bessell \& Brett 1988).

\subsection{Single Lens Model}

The PSPL model is described by the standard single-lens parameters: $t_{\mathrm{E}}$ the Einstein crossing time, $u_{o}$ the minimum impact parameter, and $t_{o}$ the time of this minimum. The 

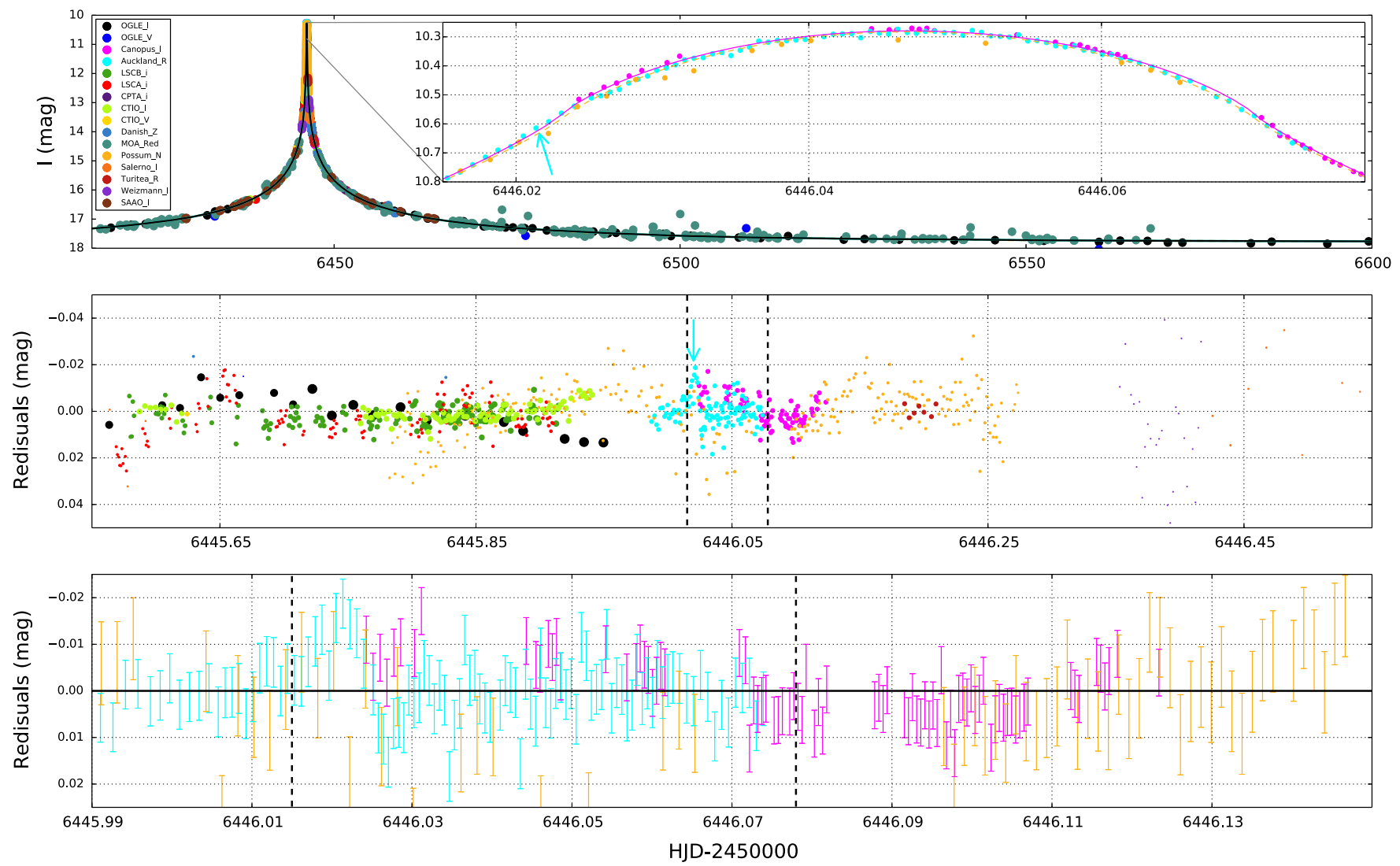

Figure 1. Light curve of OGLE-2013-BLG-0446 with our best FSPL model. The top panel shows the full 2013 light curve with a maximum magnification at HJD $-2450000 \sim 6446.0$ days. The insert on the right is a zoom of the peak. The pink model light curve is for $\Gamma_{I}=0.46$ and the orange dashed model light curve is for $\Gamma_{V}=0.63$. The cyan model for the $R$ band is not shown for clarity. The cyan arrow indicates the position of the possible planetary anomaly. The middle panel shows residuals of the FSPL model close to the peak. The radius of each point is proportional to the inverse square of the error bar (bigger points have smaller error bars). The bottom panel is a closer view of the possible anomaly. For the bottom two panels, vertical dashed black lines indicate the time window corresponding to the insert in the top figure.

normalized angular source radius $\rho=\theta_{*} / \theta_{\mathrm{E}}$ (Gould 1994; Nemiroff \& Wickramasinghe 1994; Witt \& Mao 1994; Bennett $\&$ Rhie 1996; Vermaak 2000), where $\theta_{\mathrm{E}}$ is the angular Einstein ring radius, is included in the model along with the previous parameters to take into account finite-source effects close to the magnification peak. We used the method described in Yoo et al. (2004) to take into account the change in magnification due to the extended source. The FSPL model significantly improves the fit (see Table 3). The best FSPL model is shown in Figure 1.

Using the value of $\rho$ from the FSPL model, we are able to estimate the angular radius of the Einstein ring $\theta_{\mathrm{E}}=$ $\theta_{*} / \rho=1.57 \pm 0.1$ mas and the lens-source proper motion

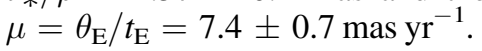

\subsection{Treatment of Photometric Uncertainties and Rejection of Outliers}

Because of the diversity of observatories and reduction pipelines used in microlensing, photometric uncertainties need careful rescaling to accurately represent the real dispersion of each data set. This is an important preliminary step in modeling the event. Following Bachelet et al. (2012b), Miyake et al. (2012), and Yee et al. (2013), we rescale the uncertainties using:

$$
e_{i}^{\prime}=\sqrt{\left(f e_{i}\right)^{2}+e_{\min }^{2}},
$$

where $e_{i}$ are the original magnitude uncertainties, $f$ is the rescaling parameter for low magnification levels, $e_{\min }$ is a minimal uncertainty to reproduce the practical limitations of photometry, and $e_{i}^{\prime}$ are the adjusted magnitude uncertainties. The classical rescaling method is to adjust $f$ and $e_{\min }$ to force $\chi^{2} /$ degrees of freedom (dof) to be unity.

In this paper, we follow an alternative method of first adjusting $f$ and $e_{\min }$ to force the residuals, normalized by $e_{i}^{\prime}$, to follow a Gaussian distribution around the model. If possible, we also aim to obtain a $\chi^{2} /$ dof $\sim 1$. Note that these two methods lead to the same results, except for the OGLE_I data set. For OGLE_I, the distribution without rescaling shows some data points with large residuals. This is not surprising because the OGLE_I data set covers the entire light curve with a large number of points, especially the faint baseline magnitude $(I \sim$ 17.8), with a constant exposure time on the order of $100 \mathrm{~s}$. Inspection of the OGLE_I light curve reveals that the uncertainties during high magnification are underestimated, so we adjust the $e_{\min }$ parameter. We tried to force $\chi^{2} /$ dof $\sim 1$ for this data set, but this generated large uncertainties for the low magnification part (i.e., the baseline), leading to a non-Gaussian distribution (lots of normalized residuals too close to the mean).

We finally checked isolated points far away from this Gaussian distribution, and reject as outliers $(>7 \sigma)$ two data points in the Auckland_R data set. The rescaling coefficients are presented in Table 2 . 
Table 2

Limg Darkening and Error Bar Rescaling Coefficients Used in this Paper

\begin{tabular}{lcccl}
\hline \hline Name & $N_{\text {data }}$ & $\Gamma_{\lambda}$ & $f$ & $e_{\text {min }}$ \\
\hline OGLE_I & 463 & 0.46 & 1.0 & 0.002 \\
OGLE_V & 24 & 0.63 & 10.25 & 0.0 \\
Canopus_I & 132 & 0.46 & 3.0 & 0.005 \\
Auckland_R & 107 & 0.55 & 1.75 & 0.005 \\
LSCB_i & 378 & 0.46 & 1.4 & 0.003 \\
LSCA_i & 385 & 0.46 & 2.0 & 0.007 \\
CPTA_i & 22 & 0.46 & 1.19 & 0.0 \\
CTIO_I & 112 & 0.46 & 1.5 & 0.004 \\
CTIO_V & 13 & 0.63 & 1.0 & 0.0 \\
Danish_z & 452 & $0.46^{\mathrm{a}}$ & 5.0 & 0.008 \\
MOA_Red & 454 & $0.51^{\mathrm{b}}$ & 1.0 & 0.0 \\
Possum_N & 244 & $0.63^{\mathrm{c}}$ & 1.5 & 0.008 \\
Salerno_I & 20 & 0.46 & 3.91 & 0.0 \\
Turitea_R & 31 & 0.55 & 1.0 & 0.005 \\
Weizmann_I & 60 & 0.46 & 3.2 & 0.01 \\
SAAO_I & 58 & 0.46 & 2.57 & 0.008 \\
\hline
\end{tabular}

Notes.

a The transmission curve for this filter is close to a Johnson Cousin I; see Skottfelt et al. (2015).

${ }^{\mathrm{b}}$ We select a bandpass between $I$ and $V$.

${ }^{\mathrm{c}}$ For this unfiltered data, we choose the filter closest to the CCD spectral response.

\subsection{Annual and Terrestrial Parallaxes}

We looked for second-order effects in the light curve. First, the relatively long Einstein-ring crossing time $\left(t_{\mathrm{E}} \sim 80\right.$ days $)$ should allow the measurement of the displacement of the line of sight toward the target due to the Earth's rotation around the Sun. This annual parallax (Gould \& Loeb 1992; Gould 2000, 2004; Smith et al. 2003; Skowron et al. 2011) is described by the vector $\pi_{\mathrm{E}, a}=A U / \widetilde{r_{\mathrm{E}}}=\left(\pi_{\mathrm{EN}}, \pi_{\mathrm{EE}}\right)$, where $\widetilde{r_{\mathrm{E}}}$ is the angular radius of the Einstein ring of the lens projected onto the observer plane and $\pi_{\mathrm{EN}}$ and $\pi_{\mathrm{EE}}$ are the components of this vector in the north and east directions respectively. In practice, the introduction of this parameter slightly changes the value of the impact parameter and $\tau=\left(t-t_{o}\right) / t_{\mathrm{E}}$. Strong modifications of the light curve can be seen far from the peak of the event, i.e., in the wings of the light curve, with few changes around the peak; see, for example, Smith et al. (2003). To model this effect, the constant $t_{o \text {,par }}$ (Skowron et al. 2011) is added to give an invariant reference time for each model. We choose $t_{o \text {, par }}=2456446.0$ HJD for our models.

Since this event is so highly magnified, it should also be possible to measure the terrestrial parallax. Hardy \& Walker (1995) first introduced the idea that for an "Extreme Microlensing Event," the difference in longitudes of observatories should result in light curves where tiny changes in the line of sight toward the target become apparent, allowing a measurement of the Einstein ring (Holz \& Wald 1996; Gould 1997; Dong et al. 2007). Again, this effect is described by the parallax vector $\pi_{\mathrm{E}, t}=\mathrm{AU} / r_{\oplus}\left(\Delta t_{o} / t_{\mathrm{E}}, \Delta U_{o}\right)$, where $r_{\oplus}$ is the Earth radius. Gould \& Yee (2013) estimated that the condition $\rho \widetilde{r_{\mathrm{E}}} \leqslant 50 r_{\oplus}$ is required to expect a measurable difference in terms of magnification. This condition leads to $\pi_{\mathrm{E}}>0.24$ for this event by using an approximate value for the normalized source star radius $\rho \sim 5 \times 10^{-4}$. A summary of longitudes and latitudes of the observatories is in Table 1 and results are summarized in Table 3.
Note that we also compute the annual parallax model for a positive impact parameter $\left(u_{0}>0\right)$ and found no significant difference with the model reported in the Table 3 . This is the $u_{0}$ degeneracy described in the literature (Smith et al. 2003; Gould 2004; Skowron et al. 2011). For the terrestrial parallax, a positive impact parameter leads to a better fit $\left(\Delta \chi^{2} \sim 60\right)$ for equivalent $\pi_{\mathrm{EN}}$ and $\pi_{\mathrm{EE}}$ values, which is a similar result to Yee et al. (2009).

\subsection{Binary Model}

At the end of the 2013 observing season, several planetary models circulated (private communication) indicating the presence of the smallest microlensing planet ever detected ( $q$ $\sim 2 \times 10^{-5}$ ). In order to investigate these claims of the existence of a very low-mass ratio planetary companion to the primary lens, and to exclude the possibility of a false alarm, we used a finite-source binary lens model (FSBL) with three extra parameters: the projected separation (normalized by $\theta_{\mathrm{E}}$ ) between the two bodies $s$, the mass ratio $q$ using the convention described in Bachelet et al. (2012a) (the most massive component on the left), and $\alpha$ the source trajectory angle measured from the line joining the two components (counterclockwise angle). We first used a grid search and we finally explore minima with a full Markov-Chain Monte Carlo algorithm. Please see, for example, Dong et al. (2009) or Bachelet et al. (2012a) for more details. We find two local minima which correspond to the known theoretical degeneracy $s \Leftrightarrow s^{-1}$. We only explore the "wide" solution, which gives the best grid-search $\chi^{2}$, for three reasons. First, as explained in the next section, the reliability of the planetary model is not clear. Second, we expect a strong degeneracy in terms of $s \Leftrightarrow s^{-1}$, so models should converge to solutions with similar shapes for the central caustic and therefore similar residuals. Finally, due to the really small value of $\rho$, modeling this event is very time consuming. We present our results in Table 3, our best causticcrossing geometry in Figure 2, and redisuals to the FSPL model for data sets covering the magnification peak are plotted in Figure 3.

\section{STUDY OF SYSTEMATIC TRENDS IN THE PHOTOMETRY}

\subsection{Generality and Method}

Our best planetary model claims the detection of smooth deviations in the light curve away from the FSPL model at a peak-to-peak level of $\leqslant 1 \%$, which is supposedly caused by the source passing over the central planetary caustic. It is well known to photometrists, however, that from ground-based telescopes the photometric precision at this level can be affected by systematic trends (or red noise) in the data.

In the early days of planet hunting using the transit method, researchers were confounded as to why they were not finding as many planets as predicted. The predictions were of course based on simulated light curves taking into account stochastic noise from the photons (sky and star) and the charge-coupled device (CCD), but ignoring the effects of sub-optimal data calibration/reduction that introduce correlated noise (e.g., Mallén-Ornelas et al. 2003 and Pepper \& Gaudi 2005). It was soon realized that transit detection thresholds were severely affected by systematic trends in the light curves (Pont et al. 2006; Aigrain \& Pont 2007) with the knock-on effect of reducing the predicted planetary yield of a transit survey, and at 
Table 3

Model Parameters

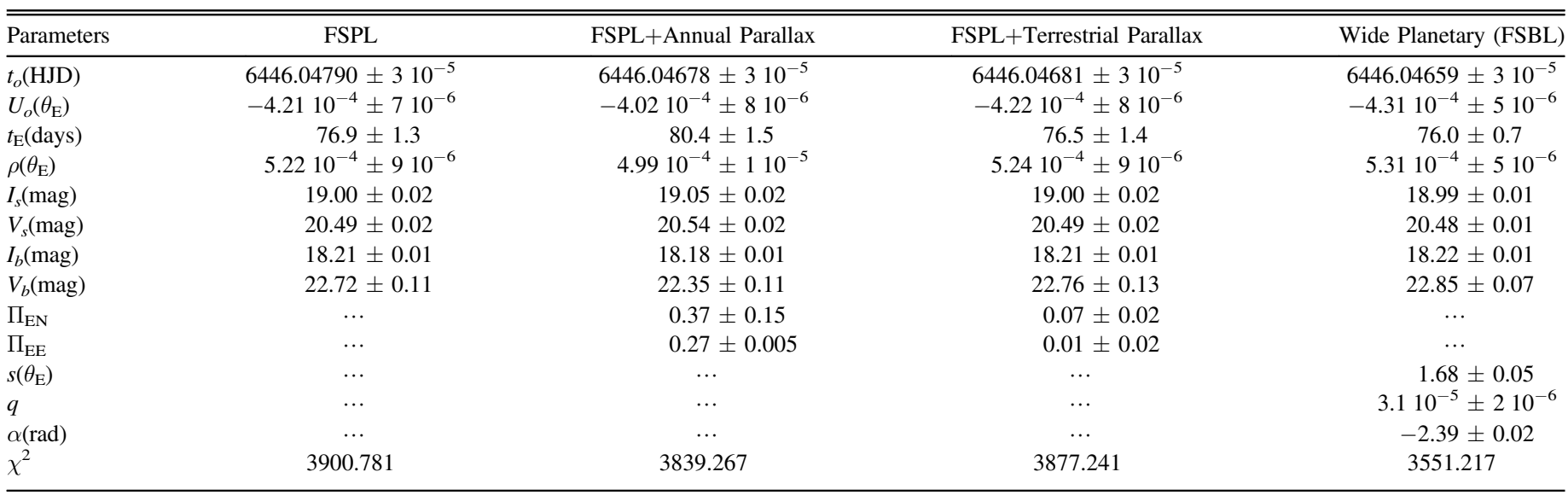

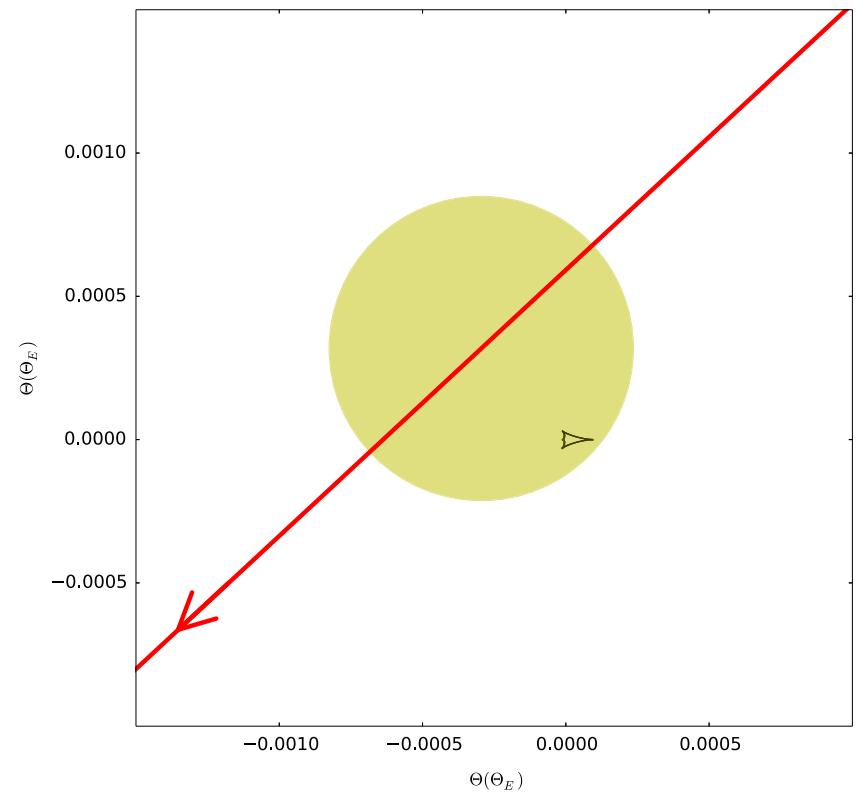

Figure 2. Lens geometry for our best fit planetary model. The yellow disk represents the source, the red line indicates the source trajectory, and the black closed curve represents the central caustic. The caustic signature in the light curve is highly "diluted" by the relatively large source star.

least partially explaining the unexpectedly low rate of transiting planet discoveries. The microlensing planet hunters face a similar problem for detecting low-amplitude $(\lesssim 1 \%)$ planetary deviations in microlensing light curves, especially when no "sharp" light curve features, caused by caustic crossing events, are predicted/observed. However, the microlensing community is now aiming for really low amplitude signal detection which requires extra care in the treatment of systematic errors (Yee et al. 2013).

Systematic trends in light curve data can be caused by an imperfect calibration of the raw data and sub-optimal extraction of the photometry. For instance, on the calibration side, flat fielding errors which vary as a function of detector coordinates can induce correlated errors in the photometry as the telescope pointing drifts slightly during a set of time-series exposures. On the software side, systematic errors in the photometry can be caused by errors in the point-spread function (PSF) model used during PSF fitting for example. Also the airmass and transparency variations in the data sets should be modeled in the DIA procedure by the photometric scale factor. However, there is no guarantee that the DIA modeling is perfect and this can create systematics trends in the data. As recently discussed by Bramich et al. (2015), an error $\epsilon_{\mathrm{p}}$ in the estimate of the photometric scale factor leads directly to an error $\epsilon_{\mathrm{p}}$ in the photometry. For example, the passage of clouds during data acquisition can create inhomogeneous atmospheric transparency in the frames and lead to a spatially varying photometric scale factor. The estimation of the photometric scale factor in DIA by using a "mean" value for the whole frame will produce different systematic trends for each star in the field of view. In practice, the expected error $\epsilon_{\mathrm{p}}$ is of order of a few per cent, which is non-critical for the majority of microlensing deviations, but can easily imitate the smallest such as in OGLE-2013-BLG-0446.

Obtaining a photon-noise limited data calibration and photometric extraction is not always feasible. Therefore complementary techniques have been developed to perform a relative calibration of the ensemble photometry after the data reduction (i.e., a post-calibration). These techniques can be divided into two broad groups, namely, detrending methods that do not use any a priori knowledge about the data acquisition or instrumental set up (e.g., Tamuz et al. 2005), and photometric modeling methods that attempt to model the systematic trends based on the survey/instrumental properties (e.g., Honeycutt 1992; Padmanabhan et al. 2008; Regnault et al. 2009). Each data point is associated with a unique object and a unique image (epoch), and carries associated metadata such as magnitude uncertainty, airmass, $(x, y)$ detector coordinates, PSF FWHM, etc. To investigate the systematic trends in the photometry, we first identified a set of object/ image properties which we suspected of having influenced the quality of the data reduction. For each of these quantities, we defined a binning that covers the full range of values with an appropriate bin size. For each bin, we introduced an unknown magnitude offset to be determined, the purpose of which is to model the mean difference of the photometric measurements within the corresponding bin from the rest of the photometric measurements. We constructed our photometric model by adopting the unknown true instrumental magnitude of each object ${ }^{63}$ and the magnitude offsets as parameters.

\footnotetext{
$\overline{63}$ Except for one object where we fixed the true instrumental magnitude to an arbitrary value to avoid degeneracy.
} 

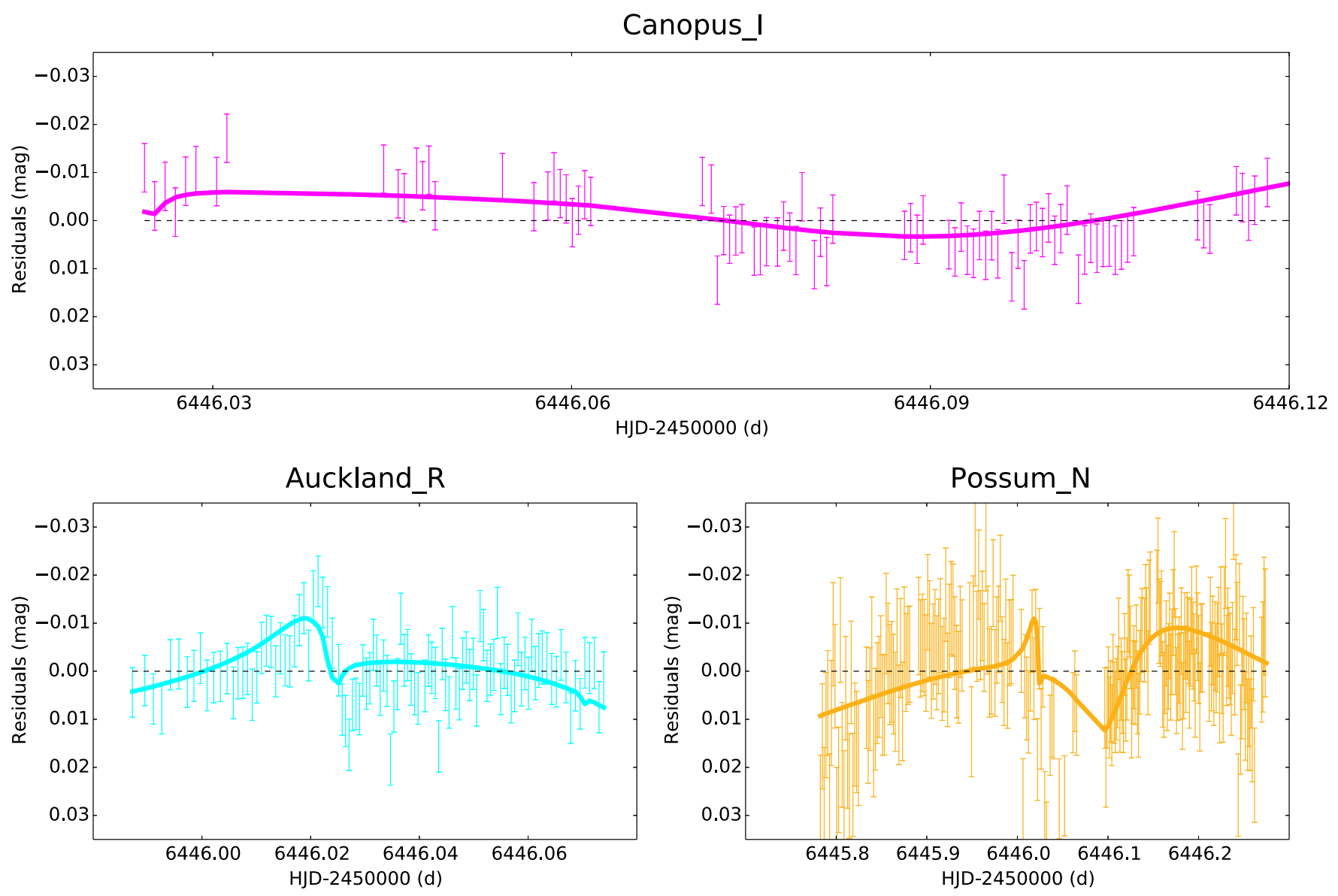

Figure 3. FSPL residuals close to the peak. The curves represent the best planetary model.

Since the model is linear, the best-fit parameter values corresponding to the minimum in $\chi^{2}$ may be solved for directly (and in a single step using some matrix algebra-see Bramich \& Freudling 2012). Iteration is of course mandatory to remove variable stars and strong outliers from the photometric data set. A valid criticism of this method is that the systematic trends are derived from the constant stars but then applied to all stars including the variable stars (the microlensing event in our case). The question arises as to whether this approach is consistent. To argue our case, we are limited to showing that the method works in practice and we direct the reader to Figure 1 of Kains et al. (2015) where RR Lyrae light curves in M68 are much improved by this self-calibration method. We used this method to analyze systematic trends in three data sets. They are listed below.

\subsection{LSCA_ $i$ and LSCB_ $i$ : the Twins Paradox}

We opted to employ the above methodology in order to investigate and understand the systematic trends in the LSCB_i and LSCA_i data sets using the algorithms described in Bramich \& Freudling (2012). ${ }^{64}$ These telescopes are twins: both are LCOGT $1 \mathrm{~m}$ telescope clones, both supporting Kodak SBIG STX-16803 CCDs at the time of these observations. SDSS- $i$ prescription filters manufactured at the same time were use to observe OGLE-2013-BLG-0446 during the same period of observation, though not precisely synchronously.

\footnotetext{
${ }^{64}$ The code is a part of DanIDL, available at http://www.danidl.co.uk/.
}

We first chose to study LSCB_i because this telescope most strongly favors the planetary model $\left(\Delta \chi^{2} \sim 128.3\right.$; see Table 6). For LSCB_i, the DanDIA pipeline extracted 4272 light curves from the images in the LSCB_i data set, each with 378 data points (or epochs), which yields a total of $1,614,816$ photometric data points. We investigated each object/image property in turn using the above method, and determined the peak-to-peak amplitude of the magnitude offsets in each case. The results are reported in Table 4. The trends in the photometry were found to be at the sub-mmag level for all correlating properties except for the epoch $(2.0 \mathrm{mmag})$. The magnitude offsets determined for each epoch (or image) serve to correct for any errors in the fitted values of the photometric scale factors during DIA. The magnitude offsets as a function of detector coordinates (commonly referred to as an illumination correction-e.g., Coccato et al. 2014) were modeled using a two-dimensional cubic surface (as opposed to the binning previously described) so as to better capture the large-scale errors in the flat-fielding. The peak-to-peak amplitude of the cubic surface over the full detector area was found to be $\sim 60$ mmag, but since the LSCB_i observations only drifted by $\sim 50$ pixels in each coordinate, we found that the magnitude offsets applicable to the OGLE-2013-BLG-446 light curve have a peak-to-peak amplitude of only $\sim 0.2 \mathrm{mmag}$. This can be seen in Figure 4. The overall level of systematic trends in the LSCB_i data set for OGLE-2013-BLG-446 is 2.0 mmag. To conclude, this analysis reveals that the illumination correction is not sufficient to explain the observed systematics. 
Table 4

The Peak-to-Peak Amplitude of the Magnitude Offsets for Each Object/Image Property that We Investigated for Causing Systematic Trends in the Photometry for the LSCB_i, LSCA_i and Auckland_R Data Sets

\begin{tabular}{|c|c|c|c|c|}
\hline \multirow[t]{2}{*}{ Correlating Quantity } & \multirow{2}{*}{ Possible Underlying Cause } & \multicolumn{3}{|c|}{ Peak-To-Peak Amplitude(mmag) } \\
\hline & & LSCA_i & LSCB_i & Auckland_R \\
\hline Exposure time & CCD non-linearities & 20 & 0.3 & 60 \\
\hline Airmass & Varying extinction & 22 & 0.8 & 40 \\
\hline PSF FWHM & Varying seeing disk & 25 & 0.4 & 28 \\
\hline Photometric scale factor & Reduction quality at different transparencies & 20 & 0.2 & 40 \\
\hline Epoch & Errors in photometric scale factor & 60 & 2.0 & 120 \\
\hline Detector coordinates & Flat-field errors & 10 & 0.2 & $*$ \\
\hline Background & Reduction quality & 27 & 0.5 & 45 \\
\hline
\end{tabular}

Note. We also list a possible underlying cause for any systematic trends that are found as a function of the corresponding object/image property.
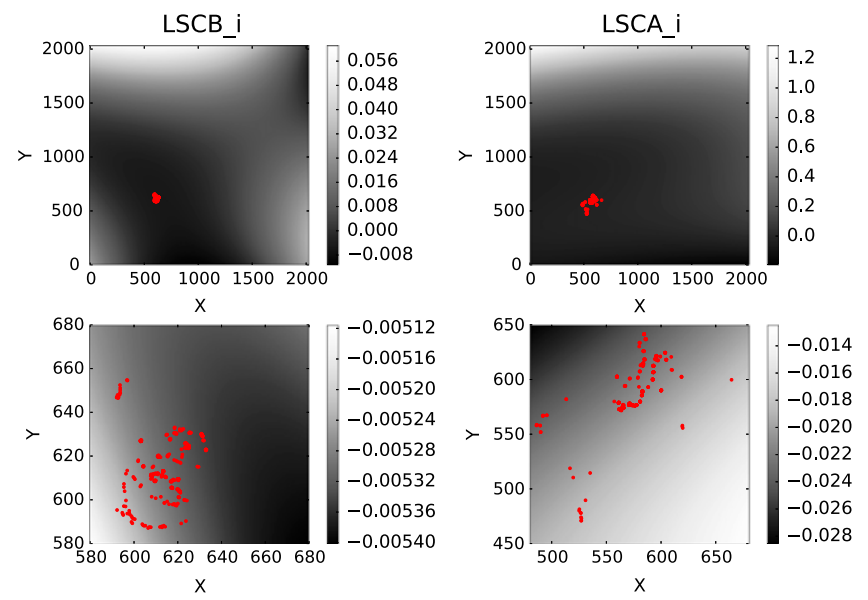

Figure 4. Illumination correction for LSCB_i and LSCA_i data sets. The bottom figures are zooms close to the pointing area.

We chose also to study the LSCA_i data set because this telescope observed the target at the same time but does not show any planetary significance $\left(\Delta \chi^{2} \sim 20.7\right)$. We conduct the same study and the results are summarized in Table 4 and Figure 4. The peak-to-peak amplitudes of the magnitude offsets are 10 times bigger than for the LSCB_i data set. It is surprising to see how two similar intruments can lead to such different data quality. A more careful check of the frames clearly shows a problem in the focus for the LSCA_i telescope. Because the Galactic Bulge fields are very crowded, this is a critical point for microlensing observations (e.g., increasing the blending). A plausible explanation for this difference between the twins is that during the time for observations, the telescopes were under commissioning, leading to non-optimal performance for LSCA_i.

\subsection{Auckland_R}

We conducted the same study for the Auckland_R data set because this telescope presents the clearest feature that mimics a planetary deviation, around HJD $\sim 6446.02$, as can be seen in Figure 3. Results can be seen in Table 4. Because the pointing for this data set was extremely accurate (offset less than 2 pixels for the whole night of observation), the estimation of the illumination correction was not possible. There is not enough information in the matrix equations and they are degenerate. But this reflects the fact that the pointing did not induce systematic trends. However, a clear variation in the magnitude offset at each epoch is visible at the time of the deviation.
Furthermore, we find that this offset is stronger for the brighter stars, as can be seen in Figure 5. There are strong similarities between the FSPL residuals and the magnitudes of the two brightest stars around the time of the anomaly HJD 6446.02, especially when the FSPL residuals get brighter at HJD 6446.03. Because the microlensing target is by far the brightest object in our field, we can expect that this systematics effect is probably even larger in our target. For this data set, we slightly modified our strategy by computing the offset at each epoch only for the brightest stars (mag $<18$ ) and we rejected the microlensing target from the computation. Also, as can be seen in the bottom panel of Figure 5, the photometric scale factor shows variations during the night. This indicates the passage of clouds which can lead to systematics errors, as described previously. For example, the FSPL residuals in the interval $6446.01 \leqslant \mathrm{HJD} \leqslant 6446.02$ clearly share the pattern with the photometric scale factor.

\subsection{Correction of Systematics}

For the three studied data sets (LSCA_i, LSCB_i and Auckland_R), we corrected the systematics for the quantity that yielded the largest peak-to-peak amplitude in the magnitude offsets: namely the epoch. Moreover, this quantity is correlated with other quantities (airmass for example) and so the epoch correction should decrease the systematic trends measured for the other paramters listed in Table 4 . We checked this and found that for the three data sets, the epoch correction leads to a significant improvement (order of a factor 10) for the systematics of the correlated quantities. This is a first order correction and we wanted to see the impact on the different models we analyzed. We repeated our modeling process with these new data sets using the previous models as starting points. The results are presented in Table 5. The new Auckland_R residuals can be seen in Figure 6. After correction, the amplitude of the "anomaly" is smaller but still exists. This is probably due to the fact that the amplitude of this feature in the light curves is brightness dependent, and the microlensing target is much brighter than all of the other stars, leading to an insufficient correcxtion fot the microlensing event.

As can be seen in Table 6, the correction of the systematics has a significant impact on the LSCA_i and Auckland_R data sets, which appear to suffer the most systematics. Note also that the planetary model is more significant after systematics correction, especially for these two telescopes. Even though the planetary model changes slightly before and after correction (the new $s$ and $q$ values are outside the error bars of the uncorrected data sets model), the caustic crossing is virtually 


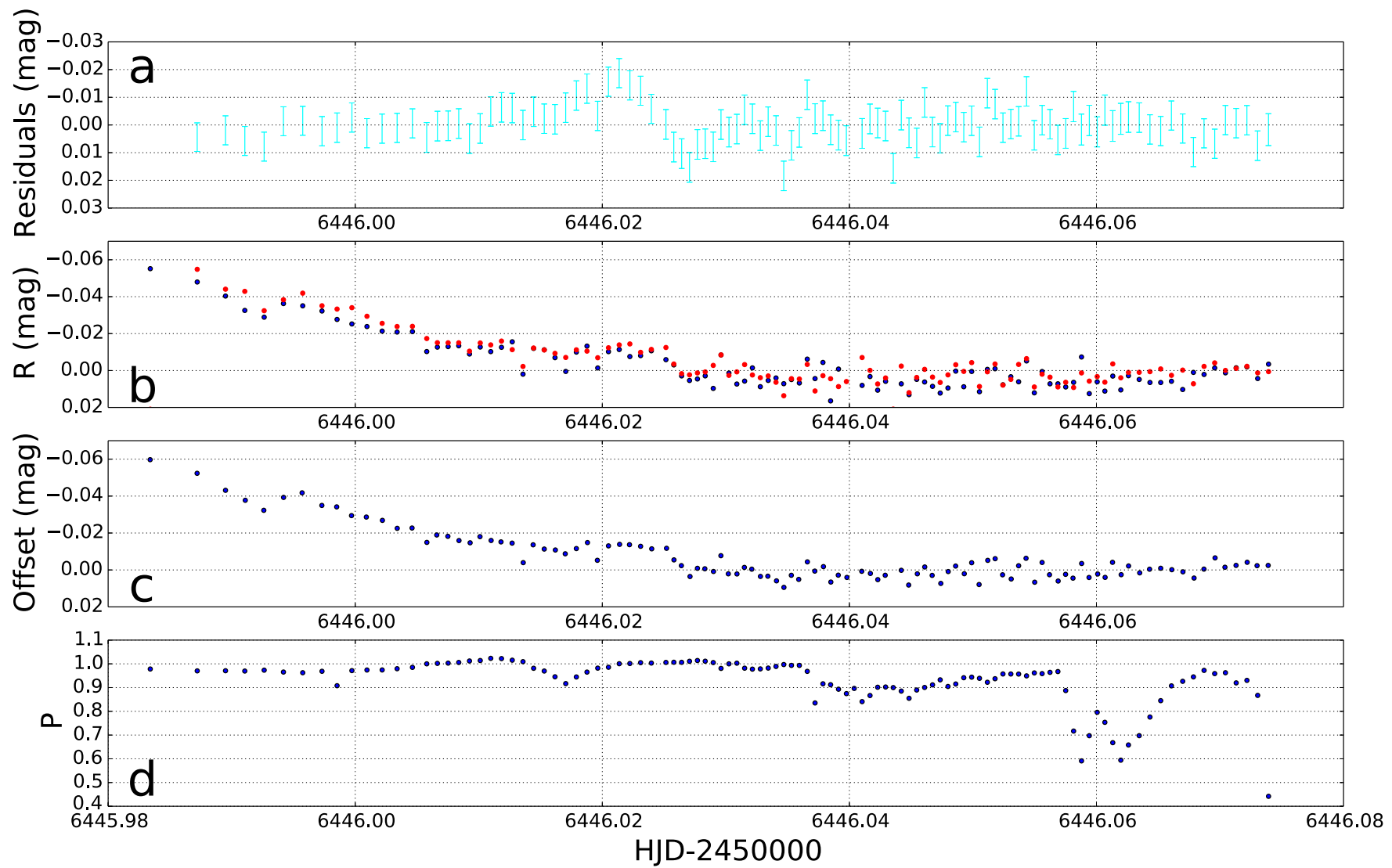

Figure 5. (a): Residuals of the FSPL model for the Auckland_R data set. (b): Light curves of the two other brightest stars in the field. (c): Systematics magnitude offsets as a function of the epoch computed for this data set; see text. (d): Photometric scale factor (normalized to a single exposure).

Table 5

Model Parameters after Correction of Systematics

\begin{tabular}{|c|c|c|c|c|}
\hline Parameters & FSPL_c & FSPL_c+Annual Parallax & FSPL_c+Terrestrial Parallax & Wide Planetary (FSBL_c) \\
\hline$\overline{t_{o}(\mathrm{HJD})}$ & $6446.04818 \pm 210^{-5}$ & $6446.04681 \pm 310^{-5}$ & $6446.04680 \pm 310^{-5}$ & $6446.04665 \pm 310^{-5}$ \\
\hline$u_{\mathbf{o}}\left(\theta_{\mathrm{E}}\right)$ & $-4.2110^{-4} \pm 110^{-6}$ & $-4.0110^{-4} \pm 810^{-6}$ & $-4.1910^{-4} \pm 710^{-6}$ & $-4.3410^{-4} \pm 510^{-6}$ \\
\hline$t_{\mathrm{E}}$ (days) & $76.8 \pm 0.1$ & $80.6 \pm 1.5$ & $77.2 \pm 1.3$ & $74.9 \pm 0.9$ \\
\hline$I_{s}(\mathrm{mag})$ & $19.00 \pm 0.01$ & $19.05 \pm 0.02$ & $19.01 \pm 0.02$ & $18.97 \pm 0.01$ \\
\hline$V_{s}(\mathrm{mag})$ & $20.49 \pm 0.01$ & $20.55 \pm 0.02$ & $20.50 \pm 0.02$ & $20.46 \pm 0.01$ \\
\hline$I_{b}(\mathrm{mag})$ & $18.21 \pm 0.01$ & $18.19 \pm 0.01$ & $18.21 \pm 0.01$ & $18.23 \pm 0.01$ \\
\hline$\Pi_{\mathrm{EE}}$ & $\cdots$ & $0.28 \pm 0.04$ & $-0.00 \pm 0.01$ & $\cdots$ \\
\hline$s\left(\theta_{\mathrm{E}}\right)$ & $\cdots$ & $\cdots$ & $\cdots$ & $1.50547 \pm 0.04$ \\
\hline$q$ & $\cdots$ & $\cdots$ & $\cdots$ & $2.30410^{-5} \pm 1.910^{-6}$ \\
\hline$\alpha(\mathrm{rad})$ & $\cdots$ & $\cdots$ & $\cdots$ & $-2.39 \pm 0.02$ \\
\hline$\chi^{2}$ & 3647.999 & 3571.000 & 3625.150 & 3258.842 \\
\hline
\end{tabular}

unchanged (e.g., the central caustic is similar). However, the clearest signature of the planetary anomaly is still in the Auckland_R data set around HJD 6446.02.

\subsection{Discussion}

Due to strong finite source effects around a very small central caustic, the suspected planetary signature in OGLE2013-BLG-0446 is very small. First of all, the low $\chi^{2}$ improvement $\left(\Delta \chi^{2} \sim 350\right.$ and $\Delta \chi^{2} \sim 389$ before and after the sysematics correction respectively) of the planetary model is far from the minimum value generally adopted in microlensing for a safe detection (Yee et al. 2013). Note also that even though the caustic crossing is similar, the two planetary models are not fully equivalent. As defined by Chung et al. (2005), the $R_{c}$ parameter is the ratio of the vertical length and the horizontal length of a central caustic. This caustic parameter before and after correction is significantly different $(\sim 30 \%)$. Second, the highest $\Delta \chi^{2}$ contributor (LSCB_i) presents photometric systematics at the same level as the planetary deviations (2 versus 6 mmag). As can be seen in Figure 6, the systematics correction decreases the amplitude of the "anomaly" in the Auckland_R data set and it is therefore better fit by the planetary model. However, the increase in the FSPL residuals after $\mathrm{HJD} \sim 6446.02$ (from $1 \%$ to zero) is not 


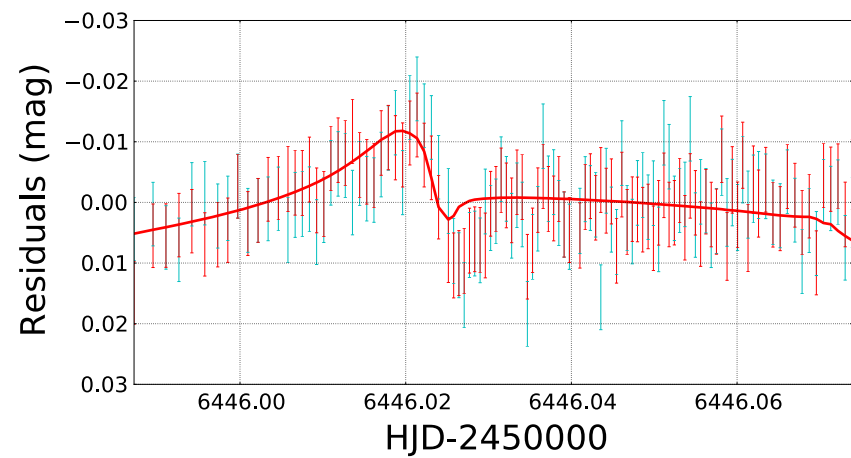

Figure 6. FSPL residuals of the Auckland_R before (cyan) and after (red) correction for systematics using the magnitude offsets as a function of epoch. The best planetary model after correction (FSBL_c) is shown in red.

explained by the planetary model, but similar behavior is seen in other bright stars. This clearly indicates that bright stars suffer from systematic effects in this data set which were not revealed by the different quantities we studied.

The planetary model is highly favored by the Canopus_I data set $\left(\Delta \chi^{2} \sim 60\right)$. However, a closer look at Figure 3 reveals that the planetary deviations are at a very low level $(\leqslant 0.5 \%)$. It cannot be excluded that the FSPL model correctly fits this data set and that this telescope also suffers from low level systematics errors. We however decided to not realize the same study of photometric systematic errors for the Canopus_I data set because there are no obvious deviations in the FSPL residuals and also because enough doubt has already been cast on the planetary model we found.

All these points reveal strong doubts about the reality of the planetary signature in OGLE-2013-BLG-0446. Even if we cannot firmly guarantee that the planet is not detected, we prefer to stay conservative and claim that we do not detect a planet in this event.

\section{CONCLUSIONS}

We presented the analysis of microlensing event OGLE2013-BLG-0446. For this highly magnified event $(A \sim 3000)$, several higher-order effects were investigated in the modeling process: annual and terrestrial parallax and planetary deviations. The study of photometric systematics for several data sets leads to various levels of confidence in the photometry. Moreover, a closer look at the data residuals and a precise study of photometric systematics reveals enough doubt to question any potential signals. Regarding the level of planetary signal $(\sim 1 \%)$ versus the various levels of systematics, we are not confident about the planetary signature in OGLE-2013-BLG0446. Unfortunately, the clearest signature of the planetary signal was observed only in a single data set which presents some unexplained behavior for the brightest stars at the time of the anomaly. These doubts in addition to the relatively low improvement in $\chi^{2}\left(\Delta \chi^{2} \leqslant 400\right)$ encourage us to remain conservative and not to claim a planetary detection. This study stresses the importance of studying and quantifying the photometric systematic errors down to the level of $1 \%$ or lower for the detecion of the smallest microlensing planets.

The authors would like to thank the anonymous referee for the usefull comments. This publication was made possible by the NPRP grant \# X-019-1-006 from the Qatar National Research Fund (a member of Qatar Foundation). The statements made herein are solely the responsibility of the authors. The authors thank the OGLE collaboration for the access of the optimized photometry. This work makes use of observations from the LCOGT network. This research has made use of the LCOGT Archive, which is operated by the California Institute of Technology, under contract with the Las Cumbres Observatory. This research has made much use of NASA's Astrophysics Data System. This research made use of the SIMBAD database, the VizieR catalog access tool, and the cross-match service provide $\mathrm{d}$ by CDS, Strasbourg, France. T.S. acknowledges the financial support from the

Table 6

$\chi^{2}$ and rms of FSPL Residuals for Each Data Set Before and After Correction of Systematics

\begin{tabular}{|c|c|c|c|c|c|c|c|c|}
\hline \multirow[t]{2}{*}{ Telescope } & \multirow{2}{*}{$\begin{array}{c}\mathrm{rms} \\
(\mathrm{mag})\end{array}$} & \multicolumn{3}{|c|}{ Raw Data } & \multirow{2}{*}{$\begin{array}{c}\mathrm{rms} \\
(\mathrm{mag})\end{array}$} & \multicolumn{3}{|c|}{ Corrected } \\
\hline & & $\chi_{\mathrm{FSPL}}^{2}$ & $\chi_{\mathrm{Pla}}^{2}$ & $\Delta \chi^{2}$ & & $\chi_{\mathrm{FSPL}}^{2}$ & $\chi_{\mathrm{Pla}}^{2}$ & $\Delta \chi^{2}$ \\
\hline OGLE_I & 0.029 & $770.308(459)$ & $655.364(456)$ & 114.944 & 0.029 & $765.677(459)$ & $661.426(456)$ & 104.251 \\
\hline OGLE_V & 1.348 & $23.998(20)$ & $24.005(17)$ & -0.007 & 1.348 & $23.998(20)$ & $23.979(17)$ & 0.019 \\
\hline Canopus_I & 0.007 & $191.996(128)$ & $132.410(125)$ & 59.586 & 0.007 & $204.40(128)$ & $137.769(125)$ & 66.631 \\
\hline Auckland_R & 0.006 & $142.839(103)$ & $128.294(100)$ & 14.545 & 0.006 & $112.917(103)$ & $72.331(100)$ & 40.586 \\
\hline LSCB_i & 0.007 & $446.170(374)$ & $\mathbf{3 1 7 . 8 8 3}(371)$ & 128.287 & 0.007 & $442.338(374)$ & 311.049(371) & 131.189 \\
\hline LSCA_i & 0.015 & $399.723(381)$ & $379.065(378)$ & 20.658 & 0.013 & $173.683(381)$ & $133.422(378)$ & 40.261 \\
\hline CPTA_i & 0.023 & 21.973(18) & $21.926(15)$ & 0.047 & 0.023 & 21.972(18) & $21.925(15)$ & 0.047 \\
\hline CTIO_I & 0.006 & $159.174(108)$ & $171.347(15)$ & -12.173 & 0.006 & $158.986(108)$ & $167.376(105)$ & -8.48 \\
\hline CTIO_V & 0.005 & $2.986(9)$ & $5.385(6)$ & -2.399 & 0.005 & $3.036(9)$ & $5.227(6)$ & -2.191 \\
\hline Danish_z & 0.017 & $448.804(448)$ & $470.960(445)$ & -22.156 & 0.017 & $450.589(448)$ & $471.301(445)$ & -20.712 \\
\hline MOA_Red & 0.785 & $727.979(450)$ & $725.116(447)$ & 2.863 & 0.783 & $727.760(450)$ & $721.763(447)$ & 5.997 \\
\hline Possum_N & 0.012 & $355.767(240)$ & $312.101(237)$ & 43.666 & 0.012 & $354.002(240)$ & $324.301(237)$ & 29.701 \\
\hline Salerno_I & 0.020 & $20.016(16)$ & $18.911(13)$ & 1.105 & 0.020 & 19.990(16) & $18.872(13)$ & 1.118 \\
\hline Turitea_R & 0.005 & $29.565(27)$ & $29.341(24)$ & 0.224 & 0.005 & $29.539(27)$ & $28.965(24)$ & 0.574 \\
\hline Weizmann_I & 0.034 & $92.383(56)$ & $92.667(53)$ & -0.284 & 0.034 & $92.389(56)$ & $92.649(53)$ & -0.260 \\
\hline SAAO_I & 0.014 & $67.100(54)$ & $66.442(51)$ & 0.658 & 0.014 & $67.085(54)$ & $66.487(51)$ & 0.598 \\
\hline Total & & 3900.781 & 3551.217 & 349.564 & & 3647.99 & 3258.842 & 389.157 \\
\hline
\end{tabular}

Note. The three corrected data sets are rendered in bold. Numbers in parentheses are the degrees of freedom for each model/data set. 
JSPS, JSPS23103002,JSPS24253004 and JSPS26247023. The M.O.A. project is supported by the grant JSPS25103508 and 23340064. D.P.B. acknowledges support from NSF grants AST-1009621 and AST-1211875, as well as NASA grants NNX12AF54G and NNX13AF64G. Work by I.A.B. and P.Y. was supported by the Marsden Fund of the Royal Society of New Zealand, contract no. MAU1104. The operation of the Danish $1.54 \mathrm{~m}$ telescope is financed by a grant to UGJ from the Danish Natural Science Research Council. We also acknowledge support from the Center of Excellence Centre for Star and Planet Formation (StarPlan) funded by The Danish National Research Foundation. The MiNDSTEp monitoring campaign is powered by ARTEMiS (Automated Terrestrial Exoplanet Microlensing Search; Dominik et al. 2008, AN 329, 248). K.A.A., M.D., K.H., M.H., C.S., R.A.S., and Y.T. are thankful to Qatar National Research Fund (QNRF), member of Qatar Foundation, for support by grant NPRP 09-476-1-078. C.S. has received funding from the European Union Seventh Framework Programme (FP7/2007-2013) under grant agreement No. 268421. Work by C.H. was supported by the Creative Research Initiative Program (2009-0081561) of National Research Foundation of Korea. S.H.G. and X.B.W. would like to thank the financial support from National Natural Science Foundation of China through grants Nos. 10873031 and 11473066 . T.C.H. acknowledges support from the Korea Research Council of Fundamental Science \& Technology (KRCF) via the KRCF Young Scientist Research Fellowship Programme and for financial support from KASI travel grant number 2013-9-40000. H.K. acknowledges the support from the European Commission under the Marie Curie IEF Programme in FP7. M.R. acknowledges support from FONDECYT postdoctoral fellowship No. 3120097. O.W. (FNRS research fellow) and J. Surdej acknowledge support from the Communauté française de Belgique-Actions de recherche concertées-Académie Wallonie-Europe. The HPC (and/or scientific visualization) resources and services used in this work were provided by the IT Research Computing group in Texas A\&M University at Qatar. IT Research Computing is funded by the Qatar Foundation for Education, Science and Community Development (http://www.qf.org.qa).

\section{REFERENCES}

Aigrain, S., \& Pont, F. 2007, MNRAS, 378, 741

Albrow, M. D., Beaulieu, J.-P., Caldwell, J. A. R., et al. 1999, ApJ, 522, 1011 Albrow, M. D., Horne, K., Bramich, D. M., et al. 2009, MNRAS, 397, 2099 Bachelet, E., Fouqué, P., Han, C., et al. 2012a, A\&A, 547, A55 Bachelet, E., Shin, I.-G., Han, C., et al. 2012b, ApJ, 754, 73 Beaulieu, J.-P., Bennett, D. P., Fouqué, P., et al. 2006, Natur, 439, 437
Bennett, D. P., Batista, V., Bond, I. A., et al. 2014, ApJ, 785, 155

Bennett, D. P., \& Rhie, S. H. 1996, ApJ, 472, 660

Bessell, M. S., \& Brett, J. M. 1988, PASP, 100, 1134

Bond, I. A., Abe, F., Dodd, R. J., et al. 2001, MNRAS, 327, 868

Bramich, D. M. 2008, MNRAS, 386, L77

Bramich, D. M., Bachelet, E., Alsubai, K. A., Mislis, D., \& Parley, N. 2015, A\&A, 577, 108

Bramich, D. M., \& Freudling, W. 2012, MNRAS, 424, 1584

Bramich, D. M., Horne, K., Albrow, M. D., et al. 2013, MNRAS, 428, 2275

Casagrande, L., Ramírez, I., Meléndez, J., Bessell, M., \& Asplund, M. 2010, A\&A, 512, A54

Chung, S.-J., Han, C., Park, B.-G., et al. 2005, ApJ, 630, 535

Claret, A. 2000, A\&A, 363, 1081

Coccato, L., Bramich, D. M., Freudling, W., \& Moehler, S. 2014, MNRAS, 438, 1256

Dominik, M., Horne, K., Allan, A., et al. 2008, AN, 329, 248

Dong, S., Bond, I. A., Gould, A., et al. 2009, ApJ, 698, 1826

Dong, S., Udalski, A., Gould, A., et al. 2007, ApJ, 664, 862

Gould, A. 1994, ApJL, 421, L71

Gould, A. 1997, ApJ, 480, 188

Gould, A. 2000, ApJ, 542, 785

Gould, A. 2004, ApJ, 606, 319

Gould, A., Dong, S., Bennett, D. P., et al. 2009, arXiv:0910.1832

Gould, A., \& Loeb, A. 1992, ApJ, 396, 104

Gould, A., Udalski, A., An, D., et al. 2006, ApJL, 644, L37

Gould, A., \& Yee, J. C. 2013, ApJ, 764, 107

Griest, K., \& Safizadeh, N. 1998, ApJ, 500, 37

Hardy, S. J., \& Walker, M. A. 1995, MNRAS, 276, L79

Holz, D. E., \& Wald, R. M. 1996, ApJ, 471, 64

Honeycutt, R. K. 1992, PASP, 104, 435

Kains, N., Arellano Ferrro, A., Figuera Jaimes, R., et al. 2015, A\&A, 578, 128

Kervella, P., \& Fouqué, P. 2008, A\&A, 491, 855

Kovács, G., Bakos, G., \& Noyes, R. W. 2005, MNRAS, 356, 557

Mallén-Ornelas, G., Seager, S., Yee, H. K. C., et al. 2003, ApJ, 582, 1123

Milne, E. A. 1921, MNRAS, 81, 361

Miyake, N., Udalski, A., Sumi, T., et al. 2012, ApJ, 752, 82

Nataf, D. M., Gould, A., Fouqué, P., et al. 2013, ApJ, 769, 88

Nemiroff, R. J., \& Wickramasinghe, W. A. D. T. 1994, ApJL, 424, L21

Paczyński, B. 1986, ApJ, 304, 1

Padmanabhan, N., Schlegel, D. J., Finkbeiner, D. P., et al. 2008, ApJ, 674, 1217

Pepper, J., \& Gaudi, B. S. 2005, ApJ, 631, 581

Pont, F., Zucker, S., \& Queloz, D. 2006, MNRAS, 373, 231

Regnault, N., Conley, A., Guy, J., et al. 2009, A\&A, 506, 999

Skottfelt, J., Bramich, D. M., Hundertmark, M., et al. 2015, A\&A, 574, A54

Skowron, J., Udalski, A., Gould, A., et al. 2011, ApJ, 738, 87

Smith, J. C., Stumpe, M. C., Van Cleve, J. E., et al. 2012, PASP, 124, 1000

Smith, M. C., Mao, S., \& Paczyński, B. 2003, MNRAS, 339, 925

Tamuz, O., Mazeh, T., \& Zucker, S. 2005, MNRAS, 356, 1466

Tsapras, Y., Street, R., Horne, K., et al. 2009, AN, 330, 4

Udalski, A. 2003a, ApJ, 590, 284

Udalski, A. 2003b, AcA, 53, 291

Udalski, A., \& Szymański, M. 2015, AcA, 65, 1

Vermaak, P. 2000, MNRAS, 319, 1011

Witt, H. J., \& Mao, S. 1994, ApJ, 430, 505

Yee, J. C., Hung, L.-W., Bond, I. A., et al. 2013, ApJ, 769, 77

Yee, J. C., Udalski, A., Sumi, T., et al. 2009, ApJ, 703, 2082

Yoo, J., DePoy, D. L., Gal-Yam, A., et al. 2004, ApJ, 603, 139 